\title{
Reconstruction of the abridged cloud data with long and narrow surface by Imageware software
}

\author{
Fu Zhang, Yakun Zhang, Zhaomei Qiu, Jun Wang, Pengjun Mao \\ College of Agricultural Engineering, Henan University of Science and Technology, Luoyang, 471003, China
}

\begin{abstract}
The reason of cloud data with long and narrow surface lack was explored, and the reconstruction method was researched. In order to get the best reconstructed surface, the surface trend and the surface cloud data errors in different surface orders were compared during the process of surface reconstruction. The results showed that the reconstruction of cloud data with long and narrow surface lack was realized. The free surface was generated through sectional breakpoint cloud data which was cut by the cloud data nearby the lack cloud data. The surface of the original cloud data was obtained through the adjustment of control points of free surface. The reconstruction of cloud data with long and narrow surface lack was finished through the generation of surface to cloud data. The research will provide new methods for the defect of the cloud data to realize surface reconstruction, and the research on reconstruction of the abridged cloud data with narrow and long surface by Imageware software will provide theoretical foundation for development and application of reverse engineering.
\end{abstract}

Keywords: long and narrow surface, cloud data, reconstruction, sectional breakpoint cloud data, control points

\section{INTRODUCTION}

With the widely use of reverse modeling system in various fields, Imageware, which is one of professional software of reverse engineering, is universal used in automobile manufacturing, aerospace, consumer appliances, and computer parts design, etc. It has formidable processing of measured data, surface modeling and error detection function, can process tens of thousands to millions of cloud data. Imageware version 13 was used in the research. However, the abridged cloud data often occurs in data acquisition process of the reverse engineering for various reasons. Such as, the uniqueness and defects of the original object and the symmetrical structure of the original surface were the self-shading structures, the accuracy of the data acquisition equipment, limitations and integrity of the object. The lack of cloud data would affect the accuracy and integrity of the reverse molding. Due to the complexity of spatial structure of the object, the important structural features should be missed if cloud data with long and narrow surface lack lost. So the reconstruction of cloud with long and narrow surface lack shouldn't be neglected in the reverse engineering ${ }^{[1-3]}$.

The reconstruction of the lack cloud data has been realized by the complement method of curve differential value. First, the cross section curves were fitted by the measurement data obtained, and the curves were extended to the regions which need to be interpolated based on collection shape of surface, through the curves editing functions, such as the curve tangent extension, parabolic extension and the extension of chord direction etc. Then the lack portion was supplied by the cloud, which was obtained by the curve extended.

The reconstruction would be achieved by the complement method of curve differential value easily when the lack cloud was in a plane basically and has a low degree of spatial folding. The method was not, however, very well when it comes to the cloud with long and narrow surface lack.

\section{THE RECONSTRUCTION OF THE CLOUD DATA WITH LONG AND NARROW SURFACE LACK}

The reconstruction of cloud data with long and narrow surface lack should be based on humancomputer interaction and consider the original object's external form, and follow the method of point to surface, surface to point again. The distribution of cloud data should be analyzed carefully, especially the lack cloud data nearby. The cloud data was separated according to their features, and the cross section command was applied at the point to get the cross section point, and the free surface of the lack cloud data was constructed by using the cross section point, and the surface's spatial structure was adjusted on the basis of the structural features of the original object, and the lack cloud data was generated by the adjusted surface.

\subsection{DATA PREPROCESSING}

The cloud data may inevitably be affected by the noise pollution for the impact of the surroundings and the prototype body's characteristics during the measurement. In order to obtain the complete and correct data and provide convenient for the modeling, the preprocessing of measured data was necessary, including format 
conversion, point filtering and smoothing filters, data reduction and interpolation ${ }^{[4-5]}$.

It was the most important work that the data was divided before the reverse engineering. The data had the same sub-surface type should be gathered into groups, it is convenient for subsequent surface model reconstruction that the whole data was divided into data fields, which were represented the different surface type. As for the lack cloud data, the lack portion should be fully considered during the data divided. In this essay the lack portion data was put in the same data field, the dividing line won't get through the lack part and the data field with lack cloud data won't be separated.

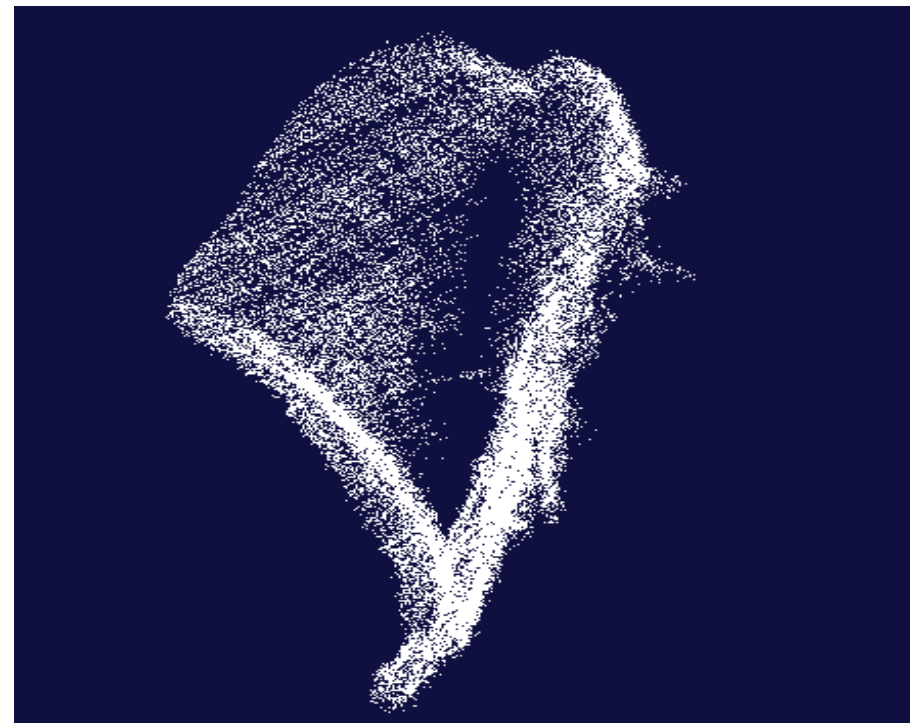

Fig.1 the divided cloud data with long and narrow surface lack

\subsection{THE RECONSTRUCTION OF LACK CLOUD DATA}

The reconstruction of the lack cloud data was realized in the following procedure.

\subsubsection{The acquisition of parallel cross section cloud data}

The profile interception was operated in order to achieve the reconstruction of cloud data with long and narrow surface lack, initially, in the isolated cloud data. The cross section and cloud parallel were selected successively in the menu of the software, and the parallel cloud cross section box was unfolded. The parallel section number was defined as 50, and the auto calculate spacing was selected, and the cloud data in the cross section was showed as Fig.2.

It is very important that the numbers will affect the quality of the reconstruction directly. The more we set the number of cross section, and the more intensive the cross section of the cloud data obtained will be, and the better continuity of the cloud data reconstructed.

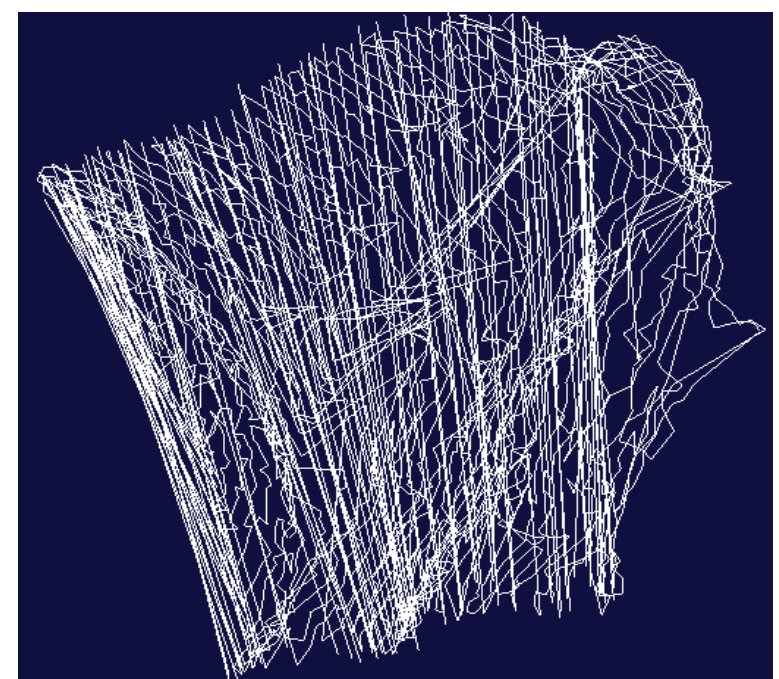

Fig.2 cloud data on the parallel sections 


\subsubsection{Free surface span selection}

The free surface was fitted by using cloud data on the parallel sections. The accuracy of the constructed cloud data largely depended on the free surface fitted. Comparing with the original objects and the original cloud data, the free surfaces fitted with different spans were analyzed. The spans of the free surface were determined ultimately. The free surface with different spans and the corresponding error were showed as Fig.3 and Fig. 4.

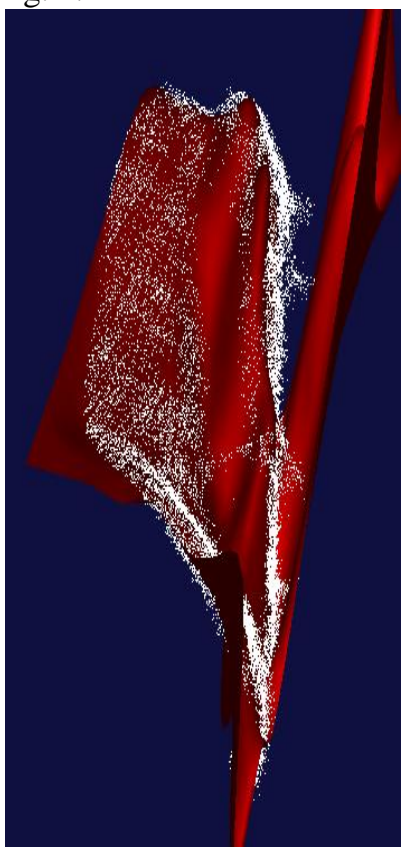

(1) Spans 10

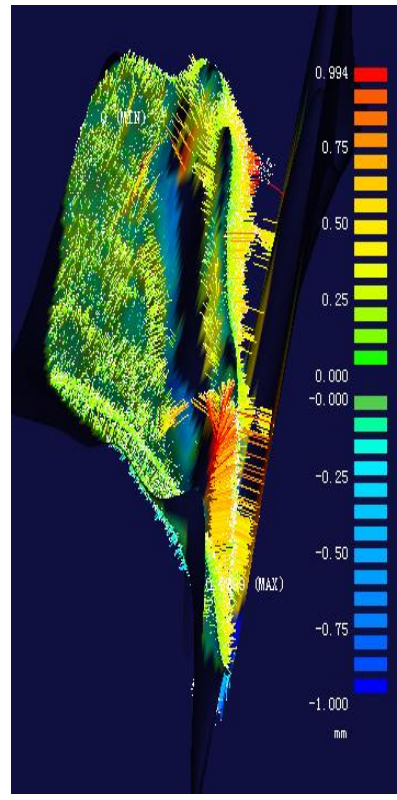

(1) Spans 10

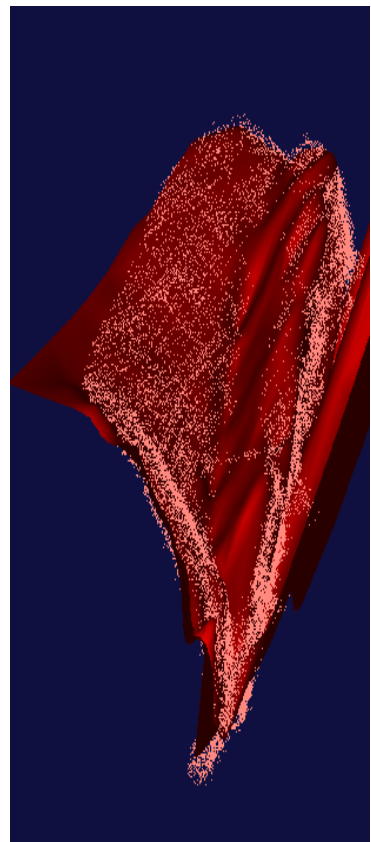

(2) Spans 15

Fig.3 free surface with different spans

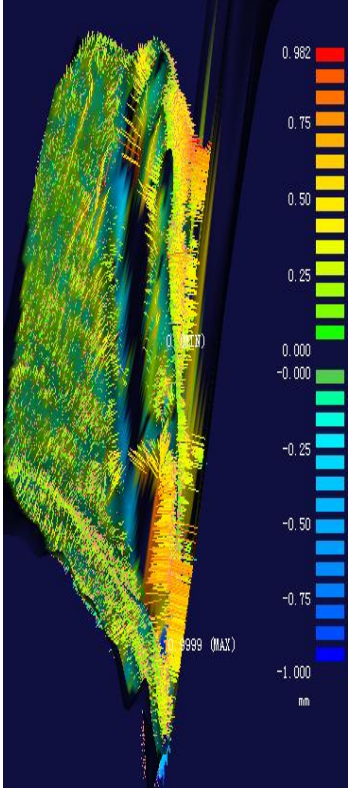

(2) Spans 15

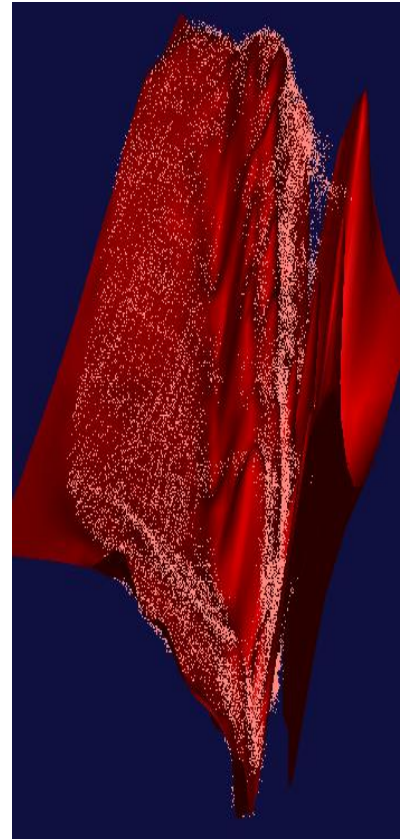

(3) Spans 20

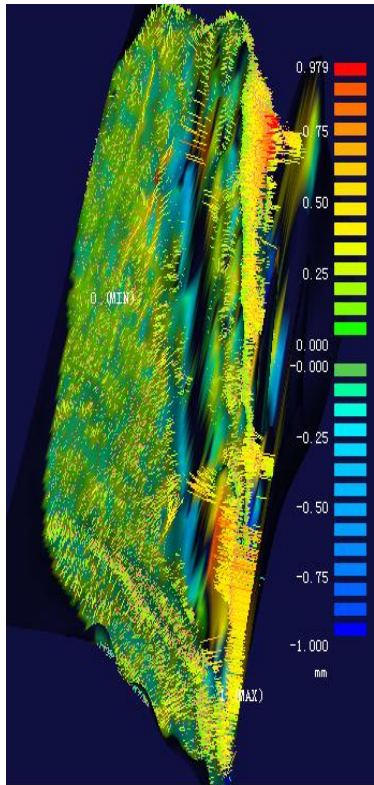

(3) Spans 20

Fig.4 error analysis of the free surface with different spans

The error analysis of the free surface with different spans showed that the spans of free surface increased from 10 to 15 , and the forward error reduces 0.012 , the percentage of the error reduction is $1 \%$. Also, the spans increased from 15 to 20 , the forward error 0.003 , the percentage $0.3 \%$. So, with the increasing spans of free surface, the error reduces gradually between the constructed free surface and the original cloud data. 
Fig.3 showed that, however, with the increase in the spans, the smoothness, which is in the right salient peak on the fitted surface, reduces gradually. There will be two salient peak generated in the right end when the spans reached to 20 , which was not consistent with the original object's entity structure as well as the original cloud data. So the free surface of spans 15 was selected to reconstruct the lack cloud data.

\subsubsection{The adjustment of free surface and cloud data generated by the free surface}

The free surface, which was showed as Fig. 5, was adjusted according to the original object's entity structure and cloud data direction. The command of surface to cloud difference must be used repeatedly when the adjustment. The cloud was reconstructed on the basis of the obtained surface, and the commands of construction, points, and sampling surface were selected successively. The cloud, which was constructed, was in Fig. 6. The long and narrow cloud data were generated based on the powerful editing command of Imageware, such as circle-select points, cloud to cloud difference. The long and narrow cloud data was showed as Fig.7.

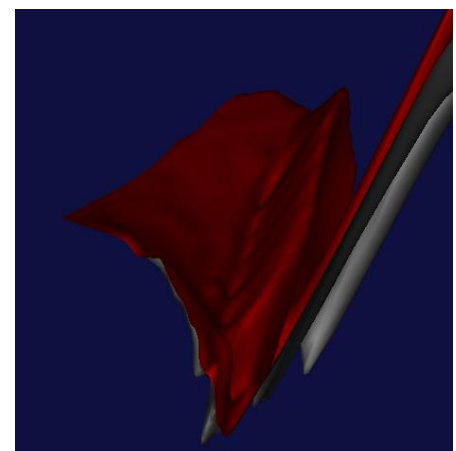

Fig.5 the adjusted free surface

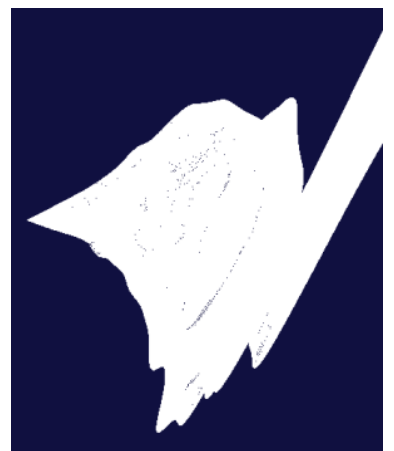

Fig.6 the generated cloud data reconstructed

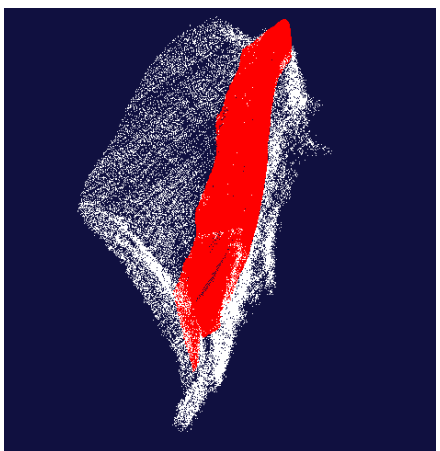

Fig.7 the long and narrow cloud data

\subsubsection{Analysis of cloud}

The cloud's errors need to be analyzed in order to evaluate the cloud with long and narrow reconstructed. The cloud difference analysis and compare were conducted between the cloud data reconstructed and the original cloud data. The results showed that the maximum error of the reconstructed cloud data was $0.3 \mathrm{~mm}$, which met the standard of reverse engineering $0.5 \mathrm{~mm}$. So the reconstruction of the cloud data with long and narrow surface lack was achieved.

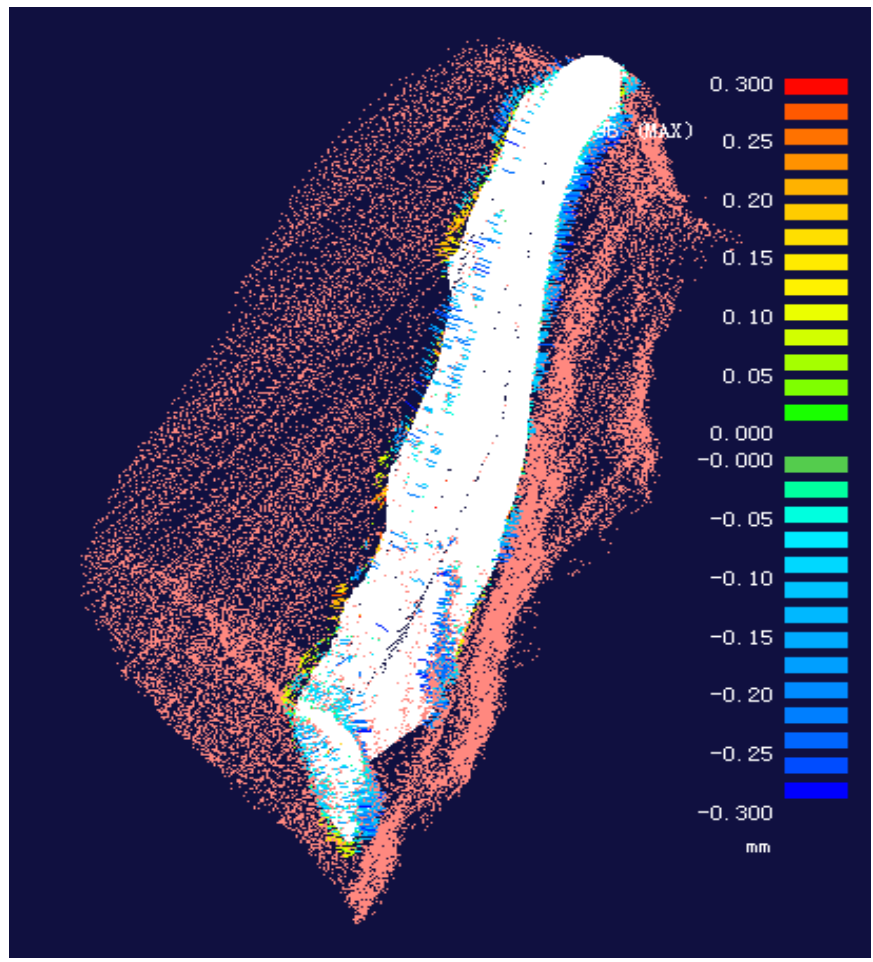

Fig.8 the color map of error analysis 
Table 1 the report of error analysis

\begin{tabular}{cccc}
\hline & Max & Average & Std. Dev \\
\hline Negative & -0.2995 & -0.1084 & 0.0777 \\
Absolute & 0.2997 & 0.1115 & 0.0749 \\
Positive & 0.2997 & 0.1143 & 0.0808 \\
\hline
\end{tabular}

\section{CONCLUSION}

This article mainly involves the reconstruction method of special cloud data, which was lack. We explore how the different spans of surface will affect the surface trend and the cloud error, and the reconstruction of cloud data with long and narrow surface lack was generated through the adjustment and the control of reconstructed surface. This method is a key supplement of 3D coordinates measurement technology, provides the important idea for the reconstruction of lack cloud data, and has the important theoretical and practical significance regarding the reverse modeling technology.

\section{ACKNOWLEDGEMENTS}

This work was supported by Natural Science Foundation of Henan Educational Committee (Grant No. 2009B210006), Scientific Research Foundation for Ph. Doctor, and Innovation Ability Foundation of Natural Science (Grant No. 2013ZCX002) of Henan University of Science and Technology.

\section{RERERENCE}

[1] Zhang Jing-tao and Lu Zhang-ping. "Surface reconstruction of the abridged cloud data in imageware". Journal of Agricultural Mechanization Research, 27(5), 2005, 90-92.

[2] Xu Shou-quan and Zhu Yan-juan. "the surface reconstruction for few and scattered points cloud". Manufacturing Information Engineering of China, 40(3), 2011, 35-44.

[3] Jin Tao and Tong Shui-guang. Reverse engineering. China Machine Press. 2003.

[4] Yu Jing-hua, Hu Zhi-yong and Hou Guo-hua. "point cloud segmentation based on imageware. Jiangsu Machine Building \& Automation”, 38(1), 2009, 113-115.

[5] Shan Yan and Xie Bin-fei. Imageware reverse modeling application examples. Tsinghua University Press. 2006. 